\title{
DESIGNING ADAPTIVE AUDIO FOR AUTONOMOUS DRIVING: AN INDUSTRIAL AND ACADEMIC-LED DESIGN CHALLENGE
}

\author{
Doon MacDonald \\ Swansea University \\ Swansea \\ SA1 8EN, Swansea, UK \\ d.g.macdonald@swansea.ac.uk
}

\begin{abstract}
The paper discusses a design challenge around the use of adaptive audio to support experience and uptake of autonomous driving. The paper outlines a collaboration that is currently being established between Researchers at Swansea university and a major OEM that is set to examine user-centred approaches to designing audio that enhance and enrich human-experience with driving.

The paper outlines the potential collaboration and describes how we will address the challenge to designing adaptive audio for unsupervised /autonomous driving. The paper outlines the research question we will address and how we will apply a tool/method that supports rapid prototyping for novice designers alongside addressing ideas around aesthetics in the interface and relationships between sound as a means for communication and as experience.
\end{abstract}

\section{INTRODUCTION}

The enthusiasm around autonomous cars is on the increase. This exciting technology has the potential to nurture positive societal changes, including reduced environmental impact, improved traffic safety and more efficient mobility. Additionally, using Autonomous Driving (AD) technology might support commuters by allowing them to be more productive to and from the commute to work (time to do work, for example). However, the introduction of highly automated cars will require a re-definition of the car-driver interaction. The ongoing technological development will put completely new demands on the design of interactions inside of the car, in order to support the driver in his or her role and to create an appropriate driving experience.

The relationship between the human and the car becomes a vital factor and is more important than ever when it comes to the trust and uptake of autonomous vehicles. The Car will be in control and so, it is only prudent to ask, what role the driver will take when the car is making the decisions? how will the driver (or, enduser) trust the car and perceive it as intelligent enough? what can we, as designers, do to enable a comfortable and safe experience for the end-user?

Even if unsupervised $\mathrm{AD}$ cars are brought to the market, their success will be down to the willingness of users to accept and

(c) (1) (8) This work is licensed under Creative Commons Attribution Non Commercial 4.0 International License. The full terms of the License are available at http://creativecommons.org/licenses/by-nc/4.0 adopt this new technology. Users need to feel that they can trust the AD technology $[1,2]$ they need to feel that it is safe to use it, they need to perceive it as being more useful than their current mode of transportation [1] and they need to enjoy using it, in order for them to accept unsupervised $\mathrm{AD}$ and eventually adopt it [2]. The user does not have to pay attention to vehicle- related visual displays any more and is freely enabled to carry out non-driving tasks [3]. This situation introduces a lot of freedom and calls for new ways of designing the user experience of the car [4].

As control is shifted away from the driver and vehicles become autonomous, there is a limit to the enjoyment felt. This is because the travelling experience for the driver is not taken into account [5]

It is fair to argue that, with the adoption of autonomous cars visible on the horizon, the car industry needs to explore important questions that focus on the human: trust, experience, up- take, acceptability and accessibility.

\section{ACADEMIA AND INDUSTRY}

Researchers from the CHERISH-Digital Economy Centre at Swansea University, UK and the OEM are developing an important relationship in order to address this challenge. Specifically, researchers at both institutions will explore the role of sound in creating a valuable user experience, with a focus on how adaptive sounds can be designed and implemented in order to support the relationship between the user and the car in a given driving scenario.

Researchers from the OEM and Swansea met at when the author presented SoundTrAD (a method and tool created by the researcher) to a driving scenario [6]. SoundTrAD is a tool that enables a designer to create prototype auditory displays and adopt a user-centred approach to the design. The tool is based on ideas and principles from Soundtrack composition. It enables a systematic approach to prototyping audio for a given scenario whereby the story and aesthetics and the use of sound as both communication and experience are important design considerations. SoundTrAD enables designers to blend different audio, test different use cases and rapidly prototype auditory displays. More is discussed on this in section 4.0.1.

A relationship was formed because the OEM and CHERISHDE both share a human-centred approach to design whereby human values remain at the heart of any technical innovation. The CHERISH-DE centre (CHERISH is an acronym for 'challenging 
Human Environments and Research Impact for a Healthy and Sustainable Digital Economy') is tasked with designing for the human, taking on human values when it comes to advancements in technology. Autonomous vehicles will be on our roads soon so together industry and academia can partner in order that the human experience is kept in the loop.

The shared design approach will take into account the needs of the user from the start of the design process and this is something that is important to both institutions. To exemplify, [7], acknowledged the need for industry and academia to work together, by observing that 'empirical Studies that follow industry projects, from design intention to the end user-experience, are scarce' [and that] 'this knowledge can enable the HCI community to better support industrial practices and aid in bridging the exisiting Industry-Academia gap'. They later go on to write that 'by cross-disciplinary research cases, we believe the discourse between industry and acdemia can be improved, and best practices for user experience can evolve'. [7]

\section{RESEARCH QUESTION AND HYPOTHESES}

There are many benefits to using Sound in the car interface. Sound, for example, can be used to provide feedback and represent information that might normally be associated with a visual channel [8]. This is particularly relevant to a potential autonomous driving scenario whereby the passenger might not be paying attention to decisions the car is making. Sound can be used for warning and alerting and sound can also represent information on many channels. Researchers are enthusiastically exploring the use of sound in $\mathrm{AD}$ as it is set to play a vital role. For example, [9] looked at spatial and multichannel audio in driverless vehicles to communicate the intended actions of the vehicle to the user. The application of Earcons and Auditory icons were explored by $[10,11]$ and specific applications were examined by [12], who looked at the use of sound to represent and warn of speed and $[13,14]$ who looked at the use of sound to represent fuel efficiency.

The specific design challenge of creating adaptive audio in relation to in-car interfaces is an area that opens up questions around the direct role of the user in a given driving scenario. Sound that can adapt in accordance to data (from the car or interaction from the user), adds the human and the car into the loop and supports the 2-way relationship between the car and the human, in as far as the real-time reaction and interaction from the user becomes an important design factor. For example, passenger information such as fatigue and levels of attention (becoming accustomed to the sound), become control variables for sound design. Furthermore, questions can be addressed concerning the emotional state and experience of the user and their subsequent trust and acceptance of the vehicle. We can design for playfulness and interaction to keep attention and engagement, or even consider the customization of the soundscape to individual people. Car information such as infotainment, other in-car noises, external sounds, external factors such as time of day, bikes, pedestrians, can all all be taken into account when it comes to the audio design for the car.

Together the OEM and Swansea University will address the following research question: Can adaptive sounds increase perceived intelligence, safety, usability and experience in unsupervised driving? The following hypotheses $(\mathrm{H})$ will be tested through a series of user-centred studies.

- H1: Adaptive Sounds can increase usability in unsupervised driving.

- H2: Adaptive sounds can enhance experience (comfort) in unsupervised driving.

- H3: Adaptive Sounds can increase a sense of intelligence and safety within the car in unsupervised driving.

\section{UPCOMING STUDY}

Collaboratively researchers will test these hypotheses. The first stage of the study will involve asking end-users to discuss their journeys to work in order to refine some use cases. This should enable the application of some real-world scenarios and refinement of parameters to work to. Following this, prototypes will be created that use adaptive sounds (effected by car data and user input) that can be tested and iterated.

Various methods and tools will be employed in order to apply and test these scenarios including:

\author{
- WOz Cars \\ - Mule Car \\ - Virtual Reality Scenario \\ - The SoundTrAD Tool (see Section 4.0.1 below)
}

\subsubsection{SoundTrAD System}

As referenced in Section 2, SoundTrAD is a tool and method that allows novice designers to prototype audio. Different sounds can be designed, blended and iterated given a specific use case. It is based on principles from Soundtrack Composition and so supports aesthetic and gamification as design considerastions[6]. Technically, SoundTrAD is programmed in Max/MSP ${ }^{1}$ and Processing ${ }^{2}$ and sends information about audio events through Open Sound Control (OSC) messages ${ }^{3}$. Designers can map out a given scenario and map sounds to different events on a time line. The events can be re-ordered in real-time and sounds subsequently tested for masking and suitability. Importantly, there is potential for SoundTrAD to be integrated (or used in alignment) with Unity ${ }^{4}$ (via OSC), thus creating the potential for working with a VR driving simulation. This is something researchers hope to explore.

\section{OUTCOMES}

From this collaboration and study a set of design guidelines will be created alongside a set of prototype examples that help industry and academia further understand the role that adaptive audio can play in autonomous driving. **We hope to further nurture a meaningful industrial and academic relationship in order to address important questions around the role of the user in future autonomous cars. Furthermore, we hope to develop and apply SoundTrAD as a useful design tool.

\section{ACKNOWLEDGMENT}

The CHERISH Digital Economy Hub, Swansea University

\footnotetext{
${ }^{1}$ https://cycling74.com

${ }^{2} \mathrm{https}$ ://processing.org

${ }^{3} \mathrm{http}: / / \mathrm{cnmat}$.berkeley.edu

${ }^{4}$ https://unity.com
} 


\section{REFERENCES}

[1] S. Nordhoff, B. Van Arem, N. Merat, R. Midigan, L. Rohrort, A. Kniw, and R. Happee, "Acceptance of driverless shuttles running in an open and mixed traffic environment." in Proc. of the 12th ITS European Congress., 2017.

[2] A. Waytz, J. Haefner, and N. Epley, "The mind in the machine: Anthropomorphism increases trust in an autonomous vehicle," Journal of Experimental Psychology, vol. 52, pp. 113-117, 2014.

[3] I. W. P. F. Cars, "Autonomous vehicles for personal transport: A technology assessment."

[4] NHTSA, "Automated vehicle policy on levels of automation and considerations for research progre," https://www.nhtsa.gov/press-releases, 2013.

[5] D. Beattie, L. Baillie, M. Halvey, and R. McCall, "Maintaining a sense of copntrol in autonomous vehicles via auditory feedback," in the Fourth Workshop on the Perceptual Quality of Systems, 2013.

[6] D. MacDonald and T. Stockman, "Soundtrad, a method and tool for prototyping auditory displays: Can we apply it to an autonomous driving scenario?" in Proc. of ICAD 2018, Meeting of the International Conference on Auditory Display, 2018.

[7] I. Pettersson, L. Hylving, A. Rydström, and D. Gkouskos, "The drive for new driving interfaces: Researching a driver interface from design intent to end-user expereince," in in Proc. of NordCHI'16, 2016.

[8] M. Jeon, S. FakhrHosseini, E. Vasey, and M. Nees, "Blueprint of the auditory interactions in automated vehicles: Report on the workshop and tutorial." in Proc. of AutoUI'17, 2017.

[9] L. Baillie, M. Halvey, and R. McCall, "What's around the corner? enhancing driver awareness in autonomous vehicles via in-vehicle spatial auditory displays," in Proc. NordCHI'14. ACM, 2014.

[10] P. Larsson, A. Opperud, k. Fredriksson, and D. Västfjäll, "Emotional and behavioural response to auditory icons and earcons in driver-vehicle interfaces," in Proc. 21st International Technical Conference on Enhanced Safety of Vehicles, Germany, 2009.

[11] P. Larsson, "Tools for designing emotional auditory drivervehicle interfaces," Auditory Display, vol. 5954, pp. 1-11, 2010.

[12] J. Hammerschmidt and T. Hermann, "Slowification: An invehicle auditory display providing speed guidance through spatial panning," in Proc. of ISon 2016, 5th Interactive Sonification Workshop, 2016.

[13] M. Nees, T. Gable, M. Jeon, and B. N. Walker, "Prototype auditory displays for a fuel efficiency driver interface," in Proc. of the 20th Int. Conf. on Auditory Displays, 2014.

[14] S. Landry, D. Tascarella, M. Jeon, and S. Fakhr Hosseini, "Listen to your drive: Sonification arhitecture and stretegies for driver state peformance," in AutomotiveUI Adjunt Proceedings '16, 2016, pp. 225-228. 